\title{
Circulating matrix metalloproteinases, their inhibitors and CD147 in children with heart diseases
}

\author{
Maksimiak L. and Kotlukova N. \\ Pirogov Russian National Research Medical University
}

\begin{abstract}
This study investigated the relationship between matrix metalloproteinases
(MMPs), their tissue inhibitors (TIMPs), CD147 and echocardiographic measurements in children with congenital heart diseases (CHD), dilated cardiomyopathies (DCM) and hypertrophic cardiomyopathies (HCM).
\end{abstract}

Methods. The study included 53 children aged from 1 month to 1 year (Table 1).

Patients were derived into 3 groups:

- CHD (37 children);

- DCM (8 children);

- $\mathrm{HCM}$ (8 children).

The control group consisted of 20 healthy children.

Enzyme-linked immunosorbent assay (ELISA) determined concentration of MMP-1, MMP-2, MMP-3, MMP-9, MMP-13, TIMP-1, TIMP-4 and CD147. Echocardiographic examination included left ventricle end-diastolic diameter (LVED), left ventricle end-systolic diameter (LVES), left ventricular ejection fraction (LVEF), left ventricular fractional shortening (LVFS). 20 children from CHD group had single ventricular septal defects (VSD). The relationship between enzyme levels and VSD sizes were also analyzed.

\section{Results.}

1) In children with CHD positive correlation were found between MMP-1 activity and LVED $(r=0,34, p<0,04)$, negative correlation between MMP-3 and LVEF, LVFS $(\mathrm{r}=-0,34, \mathrm{p}<0,04)$.

2) Plasma MMP-2 activity in the VSD patients was insignificantly correlated with the VSD size $(r=0,45$, $\mathrm{p}<0,05)$.

3) Children with DCM had an increased activity of TIMP-1 in comparison with control group $(p=0,02)$.

4) In children with DCM, positive correlation were found between CD147 activity and LVES ( $r=0,76, p<0,03)$, negative correlation between CD147 and LVEF, LVFS $(\mathrm{r}=-0,81, \mathrm{p}<0,02)$.

5) In children with HCM negative correlation were found between MMP-9 and LVEF, LVFS ( $r=-0,79, p<0,03$ )
Table 1. Gender and age of patients.

\begin{tabular}{|c|c|c|c|}
\hline \multirow{2}{*}{ Group } & \multicolumn{2}{|c|}{ Gender } & $\begin{array}{l}\text { Age (month) - } \\
\text { median } \\
\text { [interquartile } \\
\text { range, IQR] }\end{array}$ \\
\hline CHD $(n=37)$ & $35 \%(n=13)$ & $65 \%(n=24)$ & $2,0[1,0-4,0]$ \\
\hline DCM $(n=8)$ & $25 \%(n=2)$ & $75 \%(n=6)$ & $5,5[3,5-9,5]$ \\
\hline HCM $(n=8)$ & $62,5 \%(n=5)$ & $37,5 \%(n=3)$ & $3,0[1,5-7,0]$ \\
\hline $\begin{array}{c}\text { Control group } \\
(n=20)\end{array}$ & $45 \%(n=9)$ & $55 \%(n=11)$ & $4,0[2,5-5,0]$ \\
\hline
\end{tabular}

Table 2. The activity of MMPs, TIMPs, CD147 in different groups.

\begin{tabular}{|c|c|c|c|c|}
\hline & \multicolumn{4}{|c|}{ Median, (interquartile range, IQR) } \\
\hline & $\begin{array}{c}\text { CHD (n = } \\
37)\end{array}$ & $\begin{array}{l}\text { DCM } \\
(n=8)\end{array}$ & $\begin{array}{c}\mathrm{HCM} \\
(\mathrm{n}=8)\end{array}$ & $\begin{array}{l}\text { Control } \\
\text { group } \\
(n=20)\end{array}$ \\
\hline $\begin{array}{c}\text { MMP-1, } \\
\text { pg/ml }\end{array}$ & $\begin{array}{c}1020(0- \\
4908)\end{array}$ & $\begin{array}{c}1287(0- \\
2146,5)\end{array}$ & $\begin{array}{c}657,5(0- \\
7349)\end{array}$ & $0(0-1284)$ \\
\hline $\begin{array}{c}\text { MMP-2, } \\
\text { ng/ml }\end{array}$ & $\begin{array}{c}128,95 \\
(107,07- \\
145,54)\end{array}$ & $\begin{array}{c}103,11 \\
(92,26- \\
113,45)\end{array}$ & $\begin{array}{c}94,78 \\
(70,33- \\
109,02)\end{array}$ & $\begin{array}{c}121,49 \\
(99,04- \\
147,27)\end{array}$ \\
\hline $\begin{array}{c}\text { MMP-3, } \\
\text { ng/ml }\end{array}$ & $\begin{array}{c}1,91(0,75- \\
3,45)\end{array}$ & $\begin{array}{c}2,92(1,92- \\
3,44)\end{array}$ & $\begin{array}{c}1,46(0,94- \\
2,23)\end{array}$ & $\begin{array}{c}2,7(1,18- \\
4,64)\end{array}$ \\
\hline $\begin{array}{c}\text { MMP-9, } \\
\text { ng/ml }\end{array}$ & $\begin{array}{c}67,91 \\
(45,03- \\
107,45)\end{array}$ & $\begin{array}{c}57,67(32,5 \\
-72,53)\end{array}$ & $\begin{array}{c}69,46 \\
(53,51- \\
75,62)\end{array}$ & $\begin{array}{c}67,14 \\
(52,49- \\
77,9)\end{array}$ \\
\hline $\begin{array}{c}\text { MMP-13, } \\
\mathrm{pg} / \mathrm{ml}\end{array}$ & $\begin{array}{c}542,86 \\
(4,26- \\
1267,35)\end{array}$ & $\begin{array}{c}428,76(3,8 \\
-2818,94)\end{array}$ & $\begin{array}{c}309,73 \\
(59,69- \\
1296,34)\end{array}$ & $\begin{array}{c}243,27 \\
(10,7- \\
3235,26)\end{array}$ \\
\hline $\begin{array}{c}\text { TIMP-1, } \\
\text { pg/ml }\end{array}$ & $\begin{array}{c}20902,9 \\
(16011,6- \\
26729,6)\end{array}$ & $\begin{array}{l}26984,95 \\
(21573- \\
32164,2)\end{array}$ & $\begin{array}{c}20367,05 \\
(14986,55- \\
29697,05)\end{array}$ & $\begin{array}{r}18501,2 \\
(13320,45- \\
21209,4)\end{array}$ \\
\hline $\begin{array}{c}\text { TIMP-4, } \\
\text { pg/ml }\end{array}$ & $0(0-0)$ & $\begin{array}{l}0(0- \\
35,65)\end{array}$ & $\begin{array}{c}2,6(0- \\
126,4)\end{array}$ & $0(0-0)$ \\
\hline $\begin{array}{c}\text { CD147, } \\
\text { pg/ml }\end{array}$ & $\begin{array}{c}1251,63 \\
(596,71- \\
1841,21)\end{array}$ & $\begin{array}{c}789,1 \\
(550,11- \\
4364,74)\end{array}$ & $\begin{array}{c}1336,21 \\
(486,13- \\
3190,03)\end{array}$ & $\begin{array}{c}1076,59 \\
(552,47- \\
3135,74)\end{array}$ \\
\hline
\end{tabular}

